MATEC Web of Conferences 11,01038 (2014)

DOI: $10.1051 /$ matecconf $/ 20141101038$

(C) Owned by the authors, published by EDP Sciences, 2014

\title{
Effet des additifs sur la structure cristalline du polyéthylène à basse densité (PEBD) irradiés par des rayonnements UV
}

\author{
T Cheloufi1, M Berranem1, A Gasmi1 et S Benaissa2 \\ 1Université Badji Mokhtar Annaba Algérie \\ 2Université d'El-tarf Algérie
}

\begin{abstract}
The effect of UV rays on the physico -chemical and mechanical properties of low density polyethylene (LDPE) and pigmented with additives in various formulations were examined. The samples were exposed to UV radiation emitted by a xenon lamp with a power of $6000 \mathrm{~W}$ at a temperature of $46^{\circ} \mathrm{C}$ for different time of exposure. A reference sample was taken for comparison. Using infrared spectroscopy FTIR and tensile test, we were able to follow the physico-chemical and mechanical structure changes. The evolution of the mechanical parameters ( elasticity, stress and elongation at rupture) and the evolution of carbonyl groups under the effect of UV radiation were discussed. The elongation at rupture decreases with the time of exposure for most of the studied formulations, except the F4 formulation (antioxidant/ stabilizer) in which there was an increase over time. Using DSC and RX, we could calculate the degree of crystallinity which gave higher values compared to other values, and that it was probably due to the added additives ( Chimassorb 81). The results showed that the additives (light stabilizers and antioxidants) with appropriate concentrations gave reliable improvements to the mechanical behavior and the physic-chemical properties of the LDPE samples exposed to UV radiation.
\end{abstract}

Résumé. L'effet des rayons U V sur les propriétés physico-chimiques et mécaniques du polyéthylène à basse densité (PEBD) et pigmenté avec des additifs dans différentes formulations ont été étudiées. Les échantillons ont été exposés au rayonnement $\mathrm{U} V$ émis par une lampe à xénon d'une puissance de $6000 \mathrm{~W}$ à une température de $46{ }^{\circ} \mathrm{C}$ pour différents temps. Un échantillon de référence a été pris pour la comparaison. A l'aide de la spectroscopie infrarouge FTIR et l'essai de traction, on a pu suivre les changements structuraux physico-chimiques et mécaniques. L'évolution des paramètres mécaniques (module d'élasticité, contrainte et allongement à la rupture) ainsi que l'évolution des groupements carbonylés sous l'effet des radiations UV sont discutées. L'allongement à la rupture diminue avec le temps d'exposition pour la plupart des formulations étudiées, sauf la formulation F4 (anti-oxydant/stabilisant) dont il y à une augmentation avec le temps. Par les RX, on a pu calculer le taux de cristallinité qui ont donné des valeurs élevées par rapport à d'autres ce qui est du probablement aux additifs ajoutés (chimassorb 81. Les résultats obtenus montrent que les additifs (stabilisants lumière et anti-oxydants) avec des concentrations appropriées donnent des améliorations fiables concernant le comportement mécanique et les propriétés physico-chimiques des échantillons du PEBD exposés au rayonnement UV.

This is an Open Access article distributed under the terms of the Creative Commons Attribution License 2.0, which permits unrestricted use, distribution, and reproduction in any medium, provided the original work is properly cited. 


\section{Introduction}

La durée en service des polymères, et plus spécifiquement des polyoléfines (exemple : polyéthylène), est déterminée par leur stabilité à l'oxydation. En effet, à la suite d'un processus anti-oxydant, la structure moléculaire du polymère change, conduisant irrémédiablement à une chute de ses propriétés d'utilisation, le critère mécanique étant le plus souvent retenu. La synthèse d'un polymère peut se faire de différentes manières : polymérisation anionique, cationique, radicalaire, en émulsion....Le point commun à ces synthèses est souvent la présence d'agents amorceurs de réaction et ou de catalyseurs, qui sont ajoutés pour augmenter la vitesse de polymérisation. Malheureusement, les polymères «purs » sont fragiles, et ils peuvent subir, sous l'action de diverses sources (chaleur, humidité, lumière...) des dégradations qui se traduisent par une modification de leur structure chimique et entraînent une altération des propriétés physiques. Ils ne peuvent donc pas être utilisés tels quels. Lors de leur élaboration, les fabricants ajoutent diverses substances qui

\section{Techniques expérimentales}

\subsection{Matériau utilisé}

Le polyéthytène à basse densité (PEBD) utilisé pour notre étude est produit à l'entreprise nationale de l'industrie pétrochimique (ENIP de Skikda), Il est commercialisé sous forme de granulés et sous le nom de BD 09 .C'est un thermoplastique semi-cristallin (taux de cristallinité peut atteindre 50\%), fabriqué par le procédé de polymérisation en suspension, le polyéthylène produit contient $0,05 \%$ de Topanol. Le polyéthylène est mélangé avec des stabilisant lumières HALS (Hindered Amine Light Stabilizer) dans différentes formulations. Le mélange est transformé et les échantillons obtenus sont ensuite exposés au rayonnement UV émis par une lampe à xénon d'une puissance de 6000 W (Xénostest, XE-6X-HC), à une température de $46^{\circ} \mathrm{C}$ pour différentes périodes d'exposition. Un échantillon de référence est pris pour la comparaison.

\subsection{Les additifs}

Les additifs utilisés sont pour la plupart d'entre eus produits par CIBA GEIGY : modifient et renforcent leurs propriétés : des anti-oxydants, permettant aux polymères de ne pas réagir en présence de l'oxygène de l'air, des agents de réticulation, qui, après réaction chimique, peuvent se lier aux chaînes $\mathrm{du}$ polymère pour former des ponts intermoléculaires. Ces agents permettent la formation d'un réseau macromoléculaire, qui confère au matériau une meilleure stabilité mécanique et thermique. D'autres additifs peuvent aussi être ajoutés, comme des plastifiants, des colorants..., en fonction des propriétés recherchées. Tous ces additifs, lors de l'élaboration du polymère, peuvent réagir avec d'autres molécules, et on retrouve dans la matrice solide des traces de ces substances, ou des produits de dégradation de celles-ci. Il est impossible de mettre en évidence tous les types d'impuretés, ni de mesurer leur quantité dans la matrice, mais il existe bien un certain désordre dans ces matériaux; Nous appellerons ce désordre de type chimique, car il est du essentiellement à des résidus liés à la synthèse du polymère.

trois types de stabilisants lumière dont deux sont des HALS (le chimassorb944LD et le tinuvin 622LD) et un qui appartient à la famille des absorbeurs UV (chimassorb81), L'irganox 245 comme antioxydant et L'irgastat 51 comme agent antistatique.

\subsection{Préparation des échantillons}

Les additifs en poudre sont dissous dans des solvants appropriés (cétone, chloroforme) dans le but d'obtenir une distribution homogène dans le PEBD, puis mélangés avec les granulés du polyéthylène. Une partie du mélange est ensuite extrudé après séchage dans l'étuve pour éliminer toute trace du solvant. Les films sont obtenu en utilisant une extrudeuse gaine soufflage mono-vis de type BANDERA 45 avec une épaisseur qui varie entre 150 et $200 \mu \mathrm{m}$. l'autre partie du mélange est consacrée aux éprouvettes destinées aux essais mécanique, les granulés sont pressés dans un moule d'épaisseur $1 \mathrm{~mm}$ entre deux plaques chauffantes d'une presse (L-PG 7615) à une pression de 160 
tonnes. Les plaques qui en résultent sont coupées selon L'ASTM D638. Les dimensions de l'éprouvette sont représentées sur la figure1. Les conditions de transformation sont identiques pour tous les échantillons. Le tableau 1 résume les différentes formulations préparées (On note bien qu'elles contiennent toutes du Topanol sauf le PE vierge).

\subsection{Irradiation}

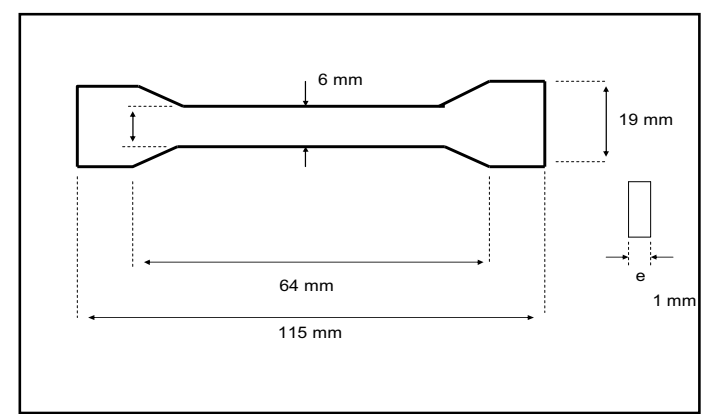

Figure 1 : Forme d'éprouvette selon L'ASTM D638
Les épaisseurs des films et des éprouvettes sont respectivement $150 \pm 5 \mu \mathrm{m}$ et $1 \pm 0.2 \mathrm{~mm}$. Ils sont placés dans le xénotest autour de la lampe à xénon de façon à recevoir toutes les radiations émises. La température de l'enceinte est environ $45 \pm 1{ }^{\circ} \mathrm{C}$, l'humidité est de $55 \%$ et les prélèvements sont effectués quatre fois après chaque cycle qui correspond à 200 heures, ce qui nous fait le total de $800 \mathrm{~h}$, y compris les échantillons

non exposés.

Tableau 1 : les différentes formulations utilisées pour l'estimation de la dégradation du PEBD

\begin{tabular}{|l|l|l|l|l|l|l|}
\hline Formulation & $\begin{array}{l}\text { Chimasso } \\
\text { rb81(\%) }\end{array}$ & $\begin{array}{l}\text { Chimassorb } \\
\mathbf{9 4 4} \mathbf{L D}(\%)\end{array}$ & $\begin{array}{l}\text { Tinuvin } \\
\mathbf{6 2 2} \mathbf{L D}(\%)\end{array}$ & $\begin{array}{l}\text { Irganox } \\
\mathbf{2 4 5}(\%)\end{array}$ & $\begin{array}{l}\text { Irgastat } \\
\mathbf{5 1}(\%)\end{array}$ & $\begin{array}{l}\text { Topanol } \\
(\%)\end{array}$ \\
\hline F0 & - & - & - & - & - & 0.05 \\
\hline F1 & 0.1 & 0.1 & - & 0.05 & 0.1 & 0.05 \\
\hline F2 & 0.2 & 0.2 & - & 0.05 & 0.1 & 0.05 \\
\hline F3 & 0.3 & 0.3 & - & 0.05 & 0.1 & 0.05 \\
\hline F4 & 0.1 & - & 0.1 & 0.05 & 0.1 & 0.05 \\
\hline F5 & 0.2 & - & 0.2 & 0.05 & 0.1 & 0.05 \\
\hline F6 & 0.3 & - & 0.3 & 0.05 & 0.1 & 0.05 \\
\hline F7 & 0.2 & - & - & 0.05 & 0.1 & 0.05 \\
\hline F8 & - & 0.2 & - & 0.05 & 0.1 & 0.05 \\
\hline F9 & - & - & 0.2 & 0.05 & 0.1 & 0.5 \\
\hline PE vierge & - & - & - & - & - & - \\
\hline
\end{tabular}




\section{CARACTERISATION PAR DES RAYONS $X$ AUX GRANDS ANGLES: WAXS}

\subsection{Appareillage}

Les analyses par diffraction des rayons $\mathrm{X}$ reposent sur la méthode de Debye Scherrer sur des films plans en transmission qui est appliquée sur un dispositif commercialisé par la société INEL. Les films plans sont numérisés à l'aide d'un scanner de marque FUJIFILM (modèle BAS 5000) et sont explorés par le logiciel TINA (Raytest). Puis, les diffractogrammes relevés à l'aide de TINA sont analysés mathématiquement à l'aide du logiciel PEAKFIT (SPSS Inc). Ainsi, l'intensité $I_{0}$ obtenue est corrigée à l'aide de la relation de Beer-Lambert qui tient compte du volume de matière absorbant en fonction de la position angulaire $2 \theta$ :

$$
I=I_{0} \cdot \exp \left(\frac{\mu \cdot e}{\cos (2 \theta)}\right)
$$

Le coefficient d'absorption $\mu=3,85 \mathrm{~cm}^{-1}$ et une épaisseur d'éprouvette $\mathrm{e}=0,08 \mathrm{~cm}$.

\subsection{Diffraction des rayons $\mathrm{X}$ aux grands angles (WAXS)}

Pour l'analyse par diffraction des rayons X, nous utilisons un dispositif expérimental commercialisé par la société INEL Le banc de diffraction possède deux modes de détection aux grands angles (compteur courbe 1D ou film plan 2D) et un mode de détection aux petits angles commercialisé par la société INEL Le banc de (film plan 2D). Le rayonnement utilisé est la raie $\mathrm{k}_{\alpha 1} 1 \mathrm{du}$ cuivre $(\lambda=0,1542 \mathrm{~nm})$. La taille $\mathrm{du}$ faisceau incident est réglée à l'aide d'un collimateur de $350 \mathrm{~m}$ de diamètre. Le rayonnement arrive normalement à la surface de l'échantillon.

La figure 2 présente le diffractogramme du film PEBD pur, dans un domaine angulaire $2 \theta, \operatorname{de} 10^{\circ}$ à $40^{\circ}$, on voit bien qu'il présente les pics de réflexion classique de la phase orthorhombique: (110), (200), pour les
Les échantillons ont une épaisseur de $0,8 \mathrm{~mm}$ et une longueur de $10 \mathrm{~mm}$. Un système de visée avec un pointeur laser permet de repérer avec précision la cible des rayons $\mathrm{X}$ sur l'échantillon. La tension et l'intensité du générateur produisant le rayonnement $\mathrm{X}$ sont respectivement $30 \mathrm{KV}$ et $40 \mathrm{~mA}$ et la durée d'exposition est de 2 heures. La position angulaire des pics de diffraction caractéristiques d'un assemblage cristallin est donnée par la loi de Bragg :

$$
2 \mathrm{~d}_{\mathrm{hkl}} \sin \theta=\mathrm{n} \lambda
$$

Où $\mathrm{d}_{\mathrm{hkl}}$ : distance inter- réticulaire

$$
\begin{aligned}
& \theta \text { : angle d'incidence (et de diffraction) } \\
& \lambda: \text { longueur d'onde du rayon incident }
\end{aligned}
$$

\subsection{L’épaisseur des lamelles}

Les pics de diffraction sont théoriquement infiniment minces lorsque le cristal a des dimensions infinies. L'élargissement des rais en dehors de celui dû au dispositif expérimental, est relié aux dimensions des cristallites.

$$
D_{h k l}=\frac{0.9 \lambda}{\delta(2 \theta) \cdot \cos \theta}
$$

valeurs $2 \theta$ égales à : $\left(21.3^{\circ}, 24.8^{\circ}\right)$. Récemment Wen-Yen Chiu et al [1], Ismail Karacan [2], A.S. Luyt et al [3], et J. Maity et al [4] ont trouvé des valeurs de $2 \theta$ égales à : $\left(21.3^{\circ}\right.$, $\mathrm{F} 0, \mathrm{~F} 1, \mathrm{~F} 2$, et F3 $\left..23 .8^{\circ}\right),\left(22^{\circ}, 24.9^{\circ}\right),\left(21.3^{\circ}\right.$, $23.6^{\circ}$ ) respectivement

\section{RESULTATS ET DISCUSSION}

\subsection{Effets des stabilisants}

\subsubsection{Cas de deux stabilisants}

- Absorbeurs UV (Chimassorb 81) avec le premier HALS (Chimassorb 944LD)

Afin de qualifier l'influence des stabilisants sur la structure et la microstructure cristalline, un 
examen des échantillons traités avec des différents stabilisants à été réalisé pour pouvoir

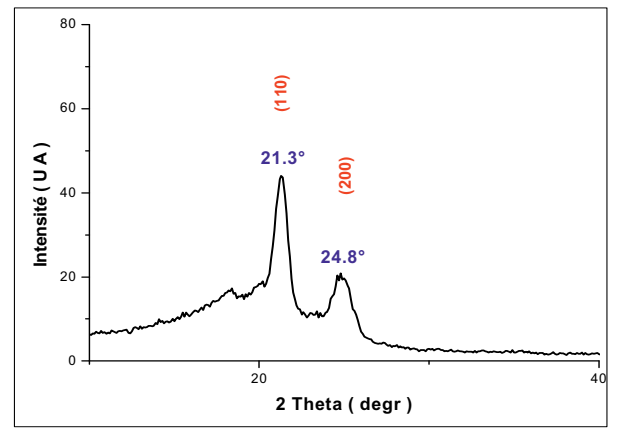

Figure 2 : Diffractogramme de rayons X (WAXS) de la formulation F0

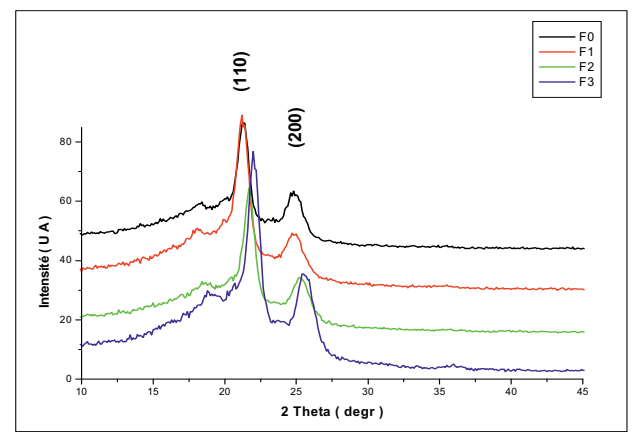

Figure 3 : Diffractogramme de rayons $\mathrm{X}$ (WAXS) des formulations F0, F1, F2, et F3

comparer l'effet des stabilisants Chimassorb 81 et Chimassorb 944LD, nous avons tracé sur la première figure (Figure 3) les spectres de diffractions RX des quatre matériaux F0,F1,F2,F3. Nous avons suivi l'évolution des

\subsubsection{Cas de chaque stabilisant}

Dans le but de suivre l'effet des stabilisants (absorbeur U V et HALS) sur la structure et la morphologie, un examen des échantillons traités avec chaque stabilisants à été réalisé. Afin de comparer les effets des stabilisants, nous avons tracé sur la même figure5 les spectres de diffraction des RX des quatre matériaux F0, F7, F8 et F9. On note que l'amplitude de l'intensité du pic (110) correspondant à F7, F8 et F9 a augmenté par rapport à celui de $\mathrm{F} 0$, ceci montre pics de réflexion (110) et (200) en fonction du pourcentage des additifs Chimassorb 81 et Chimassorb 944LD (Figure3) on voit apparaître deux pics de diffraction à $2 \theta=22^{\circ}$ et $2 \theta=25.6^{\circ}$ dont l'intensité augmente avec le pourcentage des additifs ce qui signifie que le taux de cristallisation a augmente grâce aux stabilisants et au rayonnement $\mathrm{U} \mathrm{V}$. On note aussi que le pic de diffraction (110) s'est déplacé vers les grands angles pour $\mathrm{F} 2$ et $\mathrm{F} 3$ et vers les petits angles pour F1 par rapport F0, ce qui indique que les paramètres cristallins changent après l'addition des stabilisants. Cependant la largeur à mihauteur des pics de diffraction est constante, ce qui montre que l'épaisseur moyenne des cristallites n'a pas changé après l'introduction des stabilisants.

\section{Cas d'absorbeurs UV (Chimassorb 81) avec deuxième HALS (Tinuvin 622LD)}

La figure4 montre l'évolution des pics de réflexion (110) et (200) en fonction du pourcentage des additifs Chimassorb 81 et Tinuvin 622LD, on voit les trois pics de diffractions à $2 \theta=21.2^{\circ}, 2 \theta=21.6^{\circ}$ et $2 \theta=$ $21.1^{\circ}$, dont l'intensité augmente avec le pourcentage des additifs ce qui signifie que le taux de cristallinité a augmenté grâce aux stabilisants et les rayonnements $\mathrm{U} V$. On note aussi que le pic de diffractions (110) s'est déplacé vers les grands angles pour F5 et vers les petits angles pour F4 et F6 par rapport à F0, ce qui indique que les paramètres cristallins ont changé après l'addition des stabilisants. Cependant la largeur à mi-hauteur des pics de diffraction est constante, ce qui montre que l'épaisseur moyenne des cristallites ne change pas après l'introduction des stabilisants.

que les deux types de stabilisants ont un effet sur l'évolution du taux de cristallinité. On observe aussi : L'amplitude de l'intensité du pic (110) correspondant à F7 est supérieure à celles de F8 et de F9, ce qui signifie que le taux de cristallinité a augmenté grâce à l'absorbeur UV. 


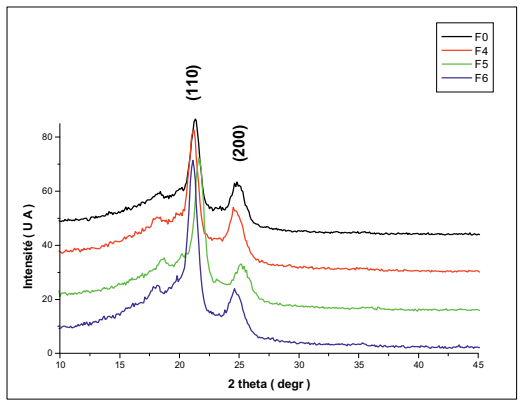

Figure 4 : Diffractogramme de rayons $X$ (WAXS) des formulations F0, F4, F5, et F6

Le pic (110) s'est déplacé vers les petits angles pour les échantillons F7 et F8, par contre pour l'échantillon F9, il s'est décalé vers les grands angles, ce qui signifie que les paramètres cristallins ont changé.

Tableau 3 : Eépaisseur de la lamelle cristallite $\mathrm{D}_{110}$ et $\mathrm{D}_{110}$

\begin{tabular}{|l|l|l|l|l|}
\hline $\begin{array}{l}\text { Formula } \\
\text { tion }\end{array}$ & $\mathbf{2 \theta _ { 1 }}\left(^{\circ}\right)$ & $\mathbf{\delta}\left(\mathbf{2} \theta_{1}\right)\left(^{\circ}\right)$ & $\mathbf{D}_{110}(\mathbf{n m})$ & $\mathbf{D}_{\mathbf{2 0 0}}(\mathbf{n m})$ \\
\hline F0 & 21.3 & 1.44 & 5.62 & 5.60 \\
\hline F1 & 22.0 & 1.39 & 5.83 & 5.88 \\
\hline F2 & 21.7 & 1.28 & 6.33 & 6.27 \\
\hline F3 & 21.2 & 1.45 & 5.58 & 5.62 \\
\hline F4 & 21.2 & 1.42 & 5.70 & 5.90 \\
\hline F5 & 21.6 & 1.43 & 5.66 & 5.57 \\
\hline F6 & 21.1 & 1.38 & 5.86 & 5.98 \\
\hline F7 & 20.3 & 1.43 & 5.65 & 6.82 \\
\hline F8 & 21.0 & 1.42 & 5.70 & 5.90 \\
\hline F9 & 22.0 & 1.31 & 6.19 & 6.43 \\
\hline
\end{tabular}

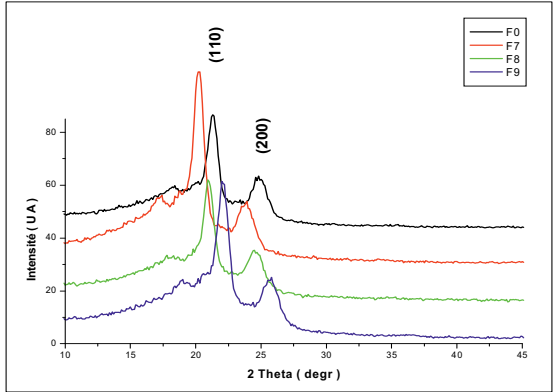

Figure 5 : Diffractogramme de rayons X (WAXS) des formulations F0, F7, F8 et F9

\section{4-2 Taux de cristallinité et dimensions moyenne des cristallites :}

A partir des diffractions des rayons $\mathrm{X}$, nous avons pu évaluer le taux de cristallinité et la dimension moyenne des cristallites perpendiculaires au plan (110), en utilisant les formules (1) et (2). Nous regroupons les valeurs du taux de cristallinité, en masse dans le tableau 2 et la dimension moyenne des cristallites dans le Tableau 3

Tableau2 : Taux de cristallinité des formulations

\begin{tabular}{|l|l|l|l|l|}
\hline $\begin{array}{l}\text { Formul } \\
\text { ation }\end{array}$ & Ac (g) & Aa (g) & At (g) & Xc(\%) \\
\hline F0 & 0.04550 & 0.08350 & 0.12900 & 35.27 \\
\hline F1 & 0.03730 & 0.06091 & 0.09821 & 37.98 \\
\hline F2 & 0.02435 & 0.04126 & 0.06561 & 37.11 \\
\hline F3 & 0.02560 & 0.04510 & 0.07070 & 36.21 \\
\hline F4 & 0.02620 & 0.03910 & 0.06530 & 40.12 \\
\hline F5 & 0.02480 & 0.04400 & 0.06880 & 36.05 \\
\hline F6 & 0.02870 & 0.04690 & 0.07560 & 37.96 \\
\hline F7 & 0.03330 & 0.05720 & 0.09050 & 36.80 \\
\hline F8 & 0.02200 & 0.03830 & 0.06030 & 36.48 \\
\hline F9 & 0.02380 & 0.05610 & 0.07990 & 29.79 \\
\hline
\end{tabular}

On voie que : Dans toutes les formulations sauf F9, le taux de cristallinité a augmenté par rapport à $\mathrm{F} 0$, ce qui signifie le rôle majeur des stabilisants sur la protection contre les rayonnements $\mathrm{U} \mathrm{V}$ [5].

Le taux de cristallinité est supérieur dans les formulations contenants deux additifs 
stabilisants (Absorbeurs U V et HALS) que dans les autres contenant un seul additif.

Le taux de cristallinité varie d'une manière linéaire avec l'augmentation de la concentration des additifs dans les formulations F1, F2 et F3 et $\mathrm{d}$ une manière aléatoire dans les formulations F4, F5 et F6. Avec une faible dose d'additif $(0.1 \%)$, on arrive à un grand taux de cristallinité $34.03 \%$ avec Chimassorb 81 et Chimassorb 944LD, dans le cas de la formulation F1, $36.33 \%$ Chimassorb 81 et Tinuvin 622LD cas de $F 4$, ce qui signifie que le pourcentage des stabilisants a une grande influence sur la morphologie cristalline du PEBD.

les formulations $\mathrm{F} 1$ et $\mathrm{F} 4$ ont des meilleurs comportements avec une faible et moyenne dose d'additif et une cristallinité acceptable, et aussi elles sont les formulations les plus adaptés d'un point de vue efficacité et coût économique.

Dans le cas d un seul stabilisant cas des échantillons F7, F8 et F9 le taux de cristallinité change d'une manière non monotone, il prend une grande valeur avec l'absorbeur U V cas de L'échantillon F7. Une légère variation de l'épaisseur des lamelles cristallines dans les deux cas $\mathrm{D}_{110}$ et $\mathrm{D}_{200}$, une augmentation de l'épaisseur des lamelles cristallines $\mathrm{D}_{110}$ pour $\mathrm{F} 2$ et F9 et pour F2, F7 et F9 dans $\mathrm{D}_{200}$. On remarque aussi que l'épaisseur des lamelles pour $\mathrm{D}_{200}$ est plus grande que celle de $\mathrm{D}_{110}$, ce qui signifie l'influence des additifs augmente vers les lamelles extérieures. Le rapport $\mathrm{D}_{200} / \mathrm{D}_{110}$ reste égal ou supérieur à un, il nous donnerons information sur le transfère de la déformation au sein des chaînes macromolécules. Dans toutes les formulations sauf $\mathrm{F} 3$, on voie l'augmentation de l'épaisseur des lamelles cristallines suivie par l'augmentation du taux de cristallinité d une manière aléatoire, on remarque aussi que les grandes valeurs du taux cristallinité se trouvent dans les formulations contenant des faibles pourcentages de deux stabilisants (Absorbeur U V et HALS), le cas de F1 et F4, et elles se trouvent aussi dans la formulation avec l'absorbeur U V, cas de F7.

\section{Conclusion}

Une corrélation entre les propriétés chimiques et physiques [11] montre une chute des propriétés mécaniques tels que l'allongement à la rupture et le module d'élasticité (E) dans les films PEBD exposés au rayonnement $\mathrm{U} \mathrm{V}$, due à la déformation des chaînes provoquées par la photo-oxydation, par contre, dans les films PEBD qui ont préparés avec des stabilisants HALS puis irradiés, les stabilisants ralentissent la vitesse des réactions de dégradation déclenchée par les radiations $U \mathrm{~V}$ et l'oxygène, et aussi en mesurant l'absorbance des principaux produits oxydés (les groupements carbonylés), les films PEBD conservent leur module d'élasticité grâce à des réactions chimiques entre les additifs et la zone amorphe, elles arrivent à freiner les déformations des chaines, ces comportement suggère que l'élasticité de PEBD est très dépendante de la concentration des stabilisants. Ce mécanisme qui concerne le processus de photo-oxydation n'est pas simple car il y a plusieurs facteurs qui l'affectent, comme l'intensité et la durée d'exposition de PE au rayonnement $\mathrm{U} V$, les impuretés, les tailles des additifs et le radical libre formé durant la synthèse des films PE utilisés. La grande efficacité de Chimassorb 81 comme photostabiliseur est due à son absorbante $\mathrm{U} \mathrm{V}$ et les réactions des groupes polaires présents à la surface avec les radicales libres formés dans le PE [12]. Comme les stabilisants se concentrent dans la phase amorphe [8], avec un mécanisme physico-chimique ces stabilisants favorisent l'augmentation du taux de cristallinité au sein de la phase amorphe. L'utilisation des photostabilisants est un facteur indispensable à la protection des films en PEBD exposés au rayonnement $\mathrm{U} \mathrm{V}$, ces additifs avec des concentrations spéciales donnent un excellent résultat concernant les propriétés mécaniques et physico-chimiques du polyéthylène irradié[1314] .

\section{References}

1. Wen-Yen Chiu, Fa-Tai Wang, Leo-Wang Chen, Trong-Ming Don, Ching-Yuan Lee Polymer degradation and stability 67, 223231 (2000)

2 Ewa Grandys, Fibres et textiles in Eastem Europe October/December, 52 (2005)

3. Luyt A.S. Geethamma V.G. , Polymer Testing 26/4 (2007) 461-470

4. Maity J, Jacob C, Das C.K, Alam S et Singh R.P Materials Science and 
Engineering, 479 (2008)

5. Ali Reza Mahdavian et al, Iranian Polymer Journal, 9 (2009)

6 Salem. M. A. et al, Macromolecular Research, 3 (2002)

7. Horrocks A.R., Mwila, J., Miraftab, M.Liu, and Chohan S.S., Polym. Deg. Stab.65, 25 (1999)

8. Frank H.P. et Lehner H., J. Polym. Sci., Part C, Symposium 31 ( 1970)

9. Salem M. A., , Egypt. J. Sol., 24 (2001)

10. Dr.Najat .J.saleh \& Zanaib.Y.shnean, Eng. \& Tech.9 (2008)

11 Alonso. M., Velasco J.I., Saja J.A European Polymer Journal, 33/3 (1997)

12. Bunn C. W., rans.Farad. oc., 35 (1954)

13. Alexander L E, New York: Wiley Interscience, (1980)

14 Chattopadhy S, Chaki T K and Bhowmick A K J. Appl. Polym. Sci. 81 (2001) 